${ }^{1} 1$ st Division of Internal Medicine, Faculty of Medicine, School of Health Sciences, University of loannina, Ioannina, Greece

2Infection Control

Committee,

UniversityHospital of Ioannina, Ioannina, Greece

${ }^{3}$ General Hospital of Corinth. Scientific Department of

Social and Educational

Policy, University of

Peloponnese, Corinth.

Hellenic Open University,

Corinth, Greece

${ }^{4}$ Division of

Gastroenterology, Faculty

of edicine, School of Health

Sciences, University of

Ioannina, Ioannina, Greece

5Pathology Laboratory,

Chair of Faculty of Medicine,

School of Health Sciences,

University of loannina,

loannina, Greece

6 Microbiology Laboratory, Faculty of Medicine,

School of Health Sciences,

University of loannina,

loannina, Greece

2nd Division of Internal Medicine, Chair of Infection Control Committee, Faculty Controlcom Sciences, University of Ioannina, Ioannina, Greece

Corresponding author: Karavasili Nicoletta, RN, BSN, ECE, MSN, MSE, PhD, Infection Control Nurse, Infection Control Committee, Infection Control Com University Hospital of loannina, loannina, Greece Ionias Ave. 12, Ioannina, Greece, PC. 45221. Fax: +30 2651099272. Mob.tel. +30 6945251997. E-mail: nicolekaravasili@gmail.com. ORCID ID: http//www.orcid. org/0000-0001-5608-5419.

\section{Clostridium Difficile Infection in Patients Impact Suspected Cytomegalovirus Infection in Patients with Inflammatory Bowel Disease}

\author{
Maria Kosmidou ${ }^{1}$, Nicoletta T. Karavasilii ${ }^{2}$, Maria Saridi ${ }^{3}$, Alexandros Skamnelos ${ }^{4}$, \\ Athanasios Kavvadias ${ }^{4}$, Anna Batistatou ${ }^{5}$, Konstantina G. Gartzonika ${ }^{6}$, Stavroula \\ Tsiara $^{7}$, Konstantinos H. Katsanos ${ }^{4}$, Dimitrios K. Christodoulou ${ }^{4}$
}

\begin{abstract}
Introduction: Clostridium difficile infection (CDI) has been reported to be a cause of flare-ups in patients with inflammatory bowel disease (IBD). Cytomegalovirus (CMV) infection can cause severe disease and complications in immunocompromised patients in consequence of disease or therapy. Aim: Our aim was to describe the prevalence and clinical outcomes of CDI with concomitant CMV infection in IBD patients hospitalized for flare-ups in association with the disease itself and medication used. Methods: We prospectively identified consecutive patients referred for CDI management during 2015-2017. Stool samples were tested for Clostridium difficile toxin A and/or B and Glutamate Dehydrogenase in patients with clinical symptoms. CDI patients with IBD history were tested for anti-CMV IgG and IgM antibodies by chemiluminescent microparticle immunoassay and underwent histological analysis for CMV on colon biopsies. Data were collected for demographic characteristics, treatment and outcome. Results: 125 patients with CDI were enrolled. Among these patients, 14 (11.2\%) were diagnosed with IBD. The mean patient age of IBD patients was $52.5 \pm 15.4$ years at diagnosis of $C D I, 85.7 \%$ had UC, $14.3 \%$ CD, while the age of patients was shared. Eleven of the total of 14 patients (78.6\%) tested positive for anti-CMV IgG. Of these, 3 patients (21.4\%) exhibited high CMV IgG avidity, without detectable anti-CMV IgM and biopsy-proven CMV colitis. Of the 14 IBD patients with CDI, 8 patients (57.1\%) were receiving anti-tumor necrosis factor (anti-TNF) therapy ( $21.4 \%$ infliximab or golimumab, $7.1 \%$ vedolizumab or adalimumab) and $43.5 \%$ of patients were being treated with systemic corticosteroids. Four UC patients (28.6\%) on steroids of the $14 \mathrm{CDI}$ patients underwent a colectomy whereas none of the not on steroids patients underwent
\end{abstract}

colectomy $(p=0.25)$. Among them, 1 patient $(7.1 \%)$ had recurrent $C D I$ after 5 months from the first episode of CDI.These patients were treated with vancomycin, metronidazole and fidaxomicin. The mean age of patients that had a colectomy $65.5 \pm 9.32(n=4)$ was higher than the mean age of those $47.30 \pm 14.49(n=10)$ who improved $\left(U_{\text {Mann-Whitney }}=6 \cdot p=0.04\right)$. Conclusions: Immunosuppressive medications and older age are associated with increased risk of CDI and poor outcome. Although, CMV is a rare colonic pathogen in the immunocompetent patient, it should be included and screened when exacerbation of IBD occurs in patients receiving any type of immunosuppressive therapy.

Keywords: Clostridium difficile, inflammatory bowel disease, cytomegalovirus, immunosuppression, anti-TNF, infectious complications.

\section{INTRODUCTION}

Inflammatory bowel disease (IBD) includes two types of idiopathic intestinal disease, ulcerative colitis (UC) and Crohn's disease (CD) that are distinguished by their location and depth of involvement in the bowel wall (1-3). The North American incidence of IBD ranges from 2.2 to 19.2 cases per 100,000 person-years for ulcerative colitis and 3.1 to 20.2 cases per 200,000 person-years for CD. IBD is much more prevalent in North America and Europe than Asia or Africa (4).

Clostridium difficile (C. difficile), a gram-positive anaerobic bacterium poses the main cause of pseudomembranous colitis $(5,6)$. Clostridium difficile infection (CDI) is a significant threat to the health of immunocompromised and hos- 
pitalized patients. Ulcerative colitis patients are at high risk for CDI (3.7\%) as well as patients with Crohn's disease (1.1\%). Most clinical trials for CDI have excluded patients with underlying diarrheal disorders such as IBD, creating an evidence gap in the management of IBD patients. The impact of CDI on these populations can be notable (7).

The diagnosis of CDI is based on the detection of toxin $\mathrm{A} / \mathrm{B}$ on stool or detection of toxin $\mathrm{A} / \mathrm{B}$ producing $\mathrm{C}$. difficile on stool by polymerase chain reaction (PCR) or culture and pseudomembrane visualization at endoscopy (8). Chronic use of antibiotics, corticosteroids and immunomodulators has been shown to increase the risk of CDI in patients with IBD $(9,10)$. CDI can alter the natural course of IBD, which is able to make it worsen and longer. It is neces $\neg$ sary for patients with IBD, hospitalized for flare signs and symptoms, the detection of opportunistic agents such as $\mathrm{C}$. difficile and cytomegalovirus or progression of the underlying IBD as causative agent of flare $(11,12)$.

Corticosteroids, immunomodulators and biologic agents used in the treatment of IBD are risk factors for CDI and CMV infection (13). CDI occurrence was more frequent in IBD patients with CMV reactivation associated with poor outcomes (14). In cases of coexisting CMV and C. difficile colitis, persistent diarrhea was not due to treatment failure for C. difficile (15).In addition, a significant role of CD4 T cells in general and Th17 cells in particular during CDI identified as a potential therapeutic target for IBD patients who are at risk for severe disease (16). IBD patients are at an increased risk of developing CDI with a poorer outcome of CDI including higher rates of colectomy and death, as well as higher rates of recurrence (17-19).

Furthermore, failure to ensure the appropriate diagnosis and treatment may lead to significant morbidity and mortality (20). Although IBD is rarely reported to be associated with $\mathrm{C}$. difficile and CMV co-infection studies have demonstrated disease exacerbations and poor outcomes in patients coinfected with C. difficile and CMV $(21,22)$.

\section{AlM}

The aim of this study was to provide observational study conducted on consecutive patients referred for CDI management to our tertiary care center, University Hospital of Ioannina, Ioannina, Greece, during 2015-2017.

\section{METHODS}

A prospective observational study was carried out during 2015-2017 in all consecutive patients with CDI.

Stool samples were tested for C. difficile toxin A and/or $B$ and Glutamate Dehydrogenase in patients with clinical symptoms. CDI was defined as a positive result on any of the following tests: C. difficile toxin A and/or B and Glutamate Dehydrogenase (C. diff quick check complete tox A/B+GDH; TechLab, Inc., Blacksburg, VA) and endoscopic findings, such as multiple yellowish plaques or whitish, consistent with pseudomembranous colitis.

CDI population with IBD history was tested for anti-CMV IgG and IgM antibodies by chemiluminescent microparticle immunoassay using a commercially available kit (CMIA; Abbott Laboratories, Abbott Park, IL). Additionally, these patients underwent histological analysis for CMV on colon
Study approval: The Institutional Review Board approved the study

A prospective observational study was carried out during 2015-2017

170.365 hospitalized patients

Our tertiary refferal care center follows 1.614 patients with IBD

125 cases with CDI: results from a single center cohort

1 patient with IBD excluded because of lack of evidence

14 cases with IBD included

Flowchart of the study

biopsies. The detection of characteristic CMV inclusions was assessed in $4 \mu \mathrm{m}$ tissue sections stained with hematoxylin-eosin. Furthermore, immunohistochemical staining was performed using monoclonal Anti-Cytomegalovirus antibody (cloneDDG9+CCH2). ). Data were collected for demographic characteristics, treatment and outcome.

\section{Study sample}

This study was a prospective observational study conducted on patients referred to our tertiary care center during 2015-2017. The population of the study treated in the University Gastroenterology Dept. and the 1st and 2nd Internal Medicine Dept. of the University Hospital of Ioannina, Greece. All patients who had been diagnosed with IBD based on clinical, endoscopic and histological criteria included. Patients underwent a complete diagnostic evaluation, which included detailed history and physical examination, serologic and stool tests in the presence of diarrhea. Exclusion criteria were colitis and diarrhea from

\begin{tabular}{|c|c|}
\hline Characteristic & C. difficile infection \\
\hline & $(n=14)$ \\
\hline Age (yr) & $52,5 \pm 15,4$ \\
\hline Male sex n (\%) & $7(50)$ \\
\hline \multicolumn{2}{|l|}{ Type of disease n (\%) } \\
\hline UC & $12(85,7)$ \\
\hline 2015 & $3(21,4)$ \\
\hline 2016 & $1(7,1)$ \\
\hline 2017 & $8(57,1)$ \\
\hline$C D$ & $2(14,3)$ \\
\hline 2015 & $0(0,0)$ \\
\hline 2016 & $1(7,1)$ \\
\hline 2017 & $1(7,1)$ \\
\hline \multicolumn{2}{|l|}{ Medication n (\%) } \\
\hline Azathioprine & $2(8,7)$ \\
\hline Mesalazine & $11(47,8)$ \\
\hline Corticosteroids & $10(43,5)$ \\
\hline \multicolumn{2}{|l|}{ Biologics n (\%) } \\
\hline Infliximab & $3(37,5)$ \\
\hline Adalimumab & $1(12,5)$ \\
\hline Vedolizumab & $1(12,5)$ \\
\hline Golimumab & $3(37,5)$ \\
\hline Recurrence rate n (\%) & $1(7,1)$ \\
\hline Colectomy rate n (\%) & $4(28,6)$ \\
\hline
\end{tabular}

Table 1. Prevalence of Clostridium difficile infection, demographics and Clinical characteristics of patients with CDI 
other causes, HIV/AIDS, cancer, multiple organ failure (flowchart).

\section{Confounding Variables and factors}

In this study potential confounders are conditions associated with secondary immunodeficiency as infectious agents (HIV/AIDS, Herpesvirus, human T-lymphotropic virus), drugs (chemotherapy), metabolic diseases (diabetes, renal failure, cirrhosis), malignancies (leukemia, lymphomas and solid tumors) and environmental conditions (radiation, heavy metals) as well as age.

Ethical compliance with human

The present study was conducted in compliance with the ethical standards of the responsible institution on human subjects as well as with the Helsinki Declaration that promotes and ensures respect for all human subjects and protects their health and rights. In particular, anonymity and confidentiality of the patients were strictly observed.

Institutional review board approval

The Institutional Review Board for University Hospital of Ioannina, Greece, approved the study. No additional permissions were required to include patient data.

\section{Statistical analysis}

All statistical analyses were performed using SPSS soft $\neg$ ware version 15.0 (SPSS Inc., Chicago, IL, USA). Continuous data were expressed as mean \pm SD, number of cases (n) and analyzed using the non parametric test Mann-Whitney Test (small samples). Categorical variables were expressed as numbers and analyzed using the chi-square or Fisher exact test. P-values $<0.05$ were considered to be sig $\neg$ nificant.

\section{RESULTS}

During the study period, 125 patients were

\begin{tabular}{|c|c|c|c|c|c|c|c|}
\hline Patient & $\begin{array}{l}\text { Age/ } \\
\text { sex }\end{array}$ & $\begin{array}{l}\text { Type of } \\
\text { disease }\end{array}$ & $\begin{array}{l}\text { Medica- } \\
\text { tion }\end{array}$ & Antibiotics & $\begin{array}{l}\text { Biolo- } \\
\text { gics }\end{array}$ & $\begin{array}{l}\text { CMV } \\
\text { biopsy }\end{array}$ & Outcome \\
\hline 1 & $38 / \mathrm{F}$ & UC & AZP & VMC, MTZ & & & Improved \\
\hline 2 & $68 / \mathrm{M}$ & UC & MSZ, CS & $\begin{array}{l}\text { CPF, VMC, MTZ, } \\
\text { MRP }\end{array}$ & IFX & - & Colectomy \\
\hline 3 & $62 / M$ & UC & CS & $\begin{array}{c}\text { VMC, MTZ, TCP } \\
\text { MRP }\end{array}$ & & - & Colectomy \\
\hline 4 & $50 / \mathrm{F}$ & $C D$ & & CPF, MTZ & IFX & - & Improved \\
\hline 5 & $43 / F$ & UC & MSZ, CS & MTZ & VDZ & & Improved \\
\hline 6 & $77 / \mathrm{F}$ & UC & MSZ, CS & CPF, VMC & GOL & - & Colectomy \\
\hline 7 & $62 \mathrm{~F}$ & UC & MSZ & VMC, MTZ & & & Improved \\
\hline 8 & $55 / \mathrm{F}$ & UC & MSZ, CS & CPF, MTZ, FDM & $\mathrm{ADA}$ & - & Colectomy \\
\hline 9 & $58 / \mathrm{F}$ & $C D$ & AZP, MSZ & VMC & & - & Improved \\
\hline 10 & $36 / \mathrm{M}$ & UC & MSZ, CS & MTZ & GOL & - & Improved \\
\hline 11 & $39 / \mathrm{M}$ & UC & MSZ, CS & VMC, MTZ & GOL & & Improved \\
\hline 12 & $21 / \mathrm{M}$ & UC & MSZ, CS & FDM & & & Improved \\
\hline 13 & $59 / M$ & UC & MSZ, CS & MTZ & IFX & & Improved \\
\hline 14 & 68/M & UC & MSZ, CS & MTZ & & - & Improved \\
\hline
\end{tabular}

Table 2. Patients with IBD and a Clostridium difficile infection suspected Cytomegalovirus infection. Azathioprine; MSZ, Mesalazine; CS, Corticosteroids; VMC, Vancomycin; MTZ, Metronidazole; CPF, Ciprofloxacin; MRP, Meropenem; TCP, Teicoplanin; FDM, Fidaxomicin; IFX, Infliximab; VDZ, Vedolizumab; ADA, Adalimumab; GOL, Golimumab.
Four UC patients $(28,6 \%)$ on steroids of the 14 IBD patients underwent a colectomy (Figure 2) whereas none of the not on steroids patients underwent colectomy $(\mathrm{p}=0.25)$. Among them, 1 patient (7,1\%) had recurrent CDI after 5 months from the first episode of CDI. These patients were treated with vancomycin, metronidazole and fidaxomicin (Table 3). The mean age of patients that had a colectomy $65.5 \pm 9.32(n=4)$ was higher than the mean age of those $47.30 \pm 14.49(\mathrm{n}=10)$ who improved (UMann-Whitney=6, $\mathrm{p}=0.04)$. The results of CDI suspected CMV infection in patients with IBD are summarized in Table 4. hospitalized because of CDI, among them; there were 14 $(11,2 \%)$ patients who were diagnosed with IBD. The mean patient age of IBD patients was $52,5 \pm 15,4$ years at diagnosis of CDI, $85,7 \%$ had UC, $14,3 \%$ CD, while the gender of patients was shared (50\% were males and $50 \%$ were females). The prevalence, demographic and clinical characteristics of patients with CDI and IBD are summarized in Table 1.

Of the 14 patients with IBD and CDI, 8 patients $(57,1 \%)$ were receiving anti-tumor necrosis factor (anti-TNF) therapy (21,4\% infliximab or golimumab, 7,1\% vedolizumab or adalimumab) and $43,5 \%$ of patients were being treated with systemic corticosteroids (Table 2).

Eleven of the total of 14 patients $(78,6 \%)$ tested positive for anti-CMV IgG (fig. 1). Of these, 3 patients $(21,4 \%)$ exhibited high CMV IgG avidity, without detectable antiCMV IgM and biopsy-proven CMV colitis.

\begin{tabular}{|c|c|c|c|c|c|c|c|c|}
\hline UC n (\%) & CD n (\%) & CDI n (\%) & $\begin{array}{l}\text { Positive anti- } \\
\text { CMV IgG (n\%) }\end{array}$ & $\begin{array}{c}\text { High CMV IgG } \\
\text { Avidity Testing } \\
\mathrm{n}(\%)\end{array}$ & $\begin{array}{c}\text { CMV biopsy } \\
\text { negative } \mathrm{n}(\%)\end{array}$ & $\begin{array}{l}\text { Steroids n } \\
\text { (\%) }\end{array}$ & $\begin{array}{c}\text { Biologics } \\
n(\%)\end{array}$ & $\begin{array}{c}\text { Colectomy } \\
\text { n (\%) }\end{array}$ \\
\hline \multirow[t]{2}{*}{$12(85,7 \%)$} & & $12(85,7 \%)$ & $9(64,3 \%)$ & $3(21,4 \%)$ & $6(42,8 \%)$ & $10(71,4 \%)$ & 7 (50\%) & $4(28,6 \%)$ \\
\hline & $2(14,3 \%)$ & $2(14,3 \%)$ & $2(14,3 \%)$ & & 2 (14,3\%) & & 1 (7,2\%) & \\
\hline
\end{tabular}

\begin{tabular}{ccccc}
\hline Outcome & $\begin{array}{c}\text { Age range } \\
\text { (frequency) } \\
(\mathrm{yr})(\mathrm{n})\end{array}$ & $\begin{array}{c}\text { CDI in patients } \\
\text { on steroids } \\
(\mathrm{n}=10)\end{array}$ & $\begin{array}{c}\text { CDI in patients } \\
\text { not on steroids } \\
(\mathrm{n}=4)\end{array}$ & $\begin{array}{c}\text { P-value } \\
\text { (Fisher's } \\
\text { exact test) }\end{array}$ \\
\hline Treatment $\mathrm{n}(\%)$ & & & & \\
\hline Vancomycin & $50-65(3)$ & $4(28,6 \%)$ & $(21,4 \%)$ & 0.55 \\
\hline Metronidazole & $<50(5)$ & $8(57,1 \%)$ & $3(21,4 \%)$ & 1.00 \\
\hline Fidaxomicin & $>65(2)$ & $2(14,3 \%)$ & $0(0,0 \%)$ & 1.00 \\
\hline Biologics $\mathrm{n}(\%)$ & & & & \\
\hline Infliximab & $50-65,>65(1)$ & $2(14,3 \%)$ & $1(7,1 \%)$ & 1.00 \\
\hline Golimumab & $<50(2)$ & $3(21,4 \%)$ & $0(0,0 \%)$ & 0.50 \\
\hline Recurrence $\mathrm{n}(\%)$ & $50-65(1)$ & $1(7,1 \%)$ & $0(0,0 \%)$ & 1.00 \\
\hline Colectomy n (\%) & $>50(4)$ & $4(28,6 \%)$ & $0(0,0 \%)$ & 0.25 \\
\hline
\end{tabular}

Table 3. Clostridium difficile infection outcome among the study group. Values are presented as number (\%), or p-value.

Table 4. The results of CDI suspected CMV infection in patients with IBD. Values are presented as number (\%). 


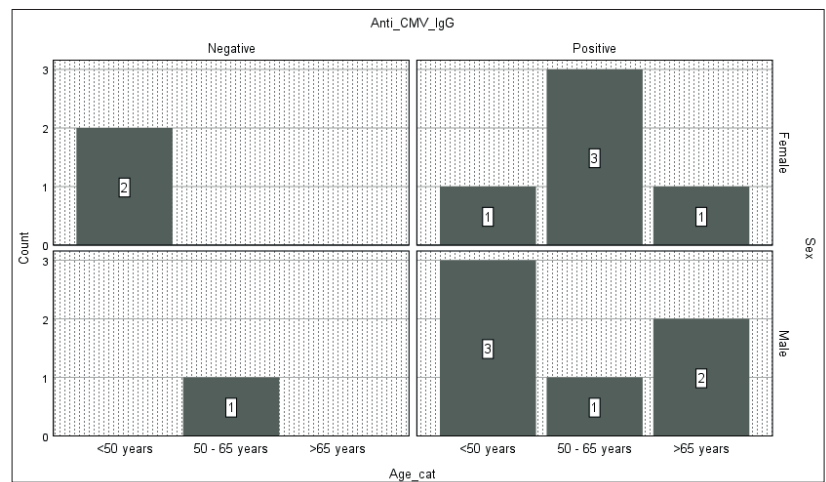

Figure 1. Patients tested for anti-CMV IgG

\section{DISCUSSION}

This study detected co-existent CDI in $14(11,2 \%)$ IBD patients of 125 patients hospitalized with CDI. This incidence of CDI in patients with IBD is relatively high considering the incidence rate of Healthcare Associated CDI in nonIBD population (67\%) (23). Our results are similar to recent studies reporting CDI incidence of $7 \%$ among hospitalized adult inpatients with IBD (24).

Furthermore, the incidence of CDI in mixed IBD populations (inpatient and outpatient) ranges between $5.1 \%$ $16.7 \%(25)$.

There is significant uncertainty among clinicians regarding the initiation of corticosteroid therapy in the setting of suspected IBD flare in a patient with known CDI. Some previous studies have reported that CDI in patients with IBD may be associated with the use of steroids describing worse outcomes among IBD patients $(26,27)$.

In this study of the 14 patients with CDI, 4 UC patients $(28,6 \%)$ underwent a colectomy, while they were treated with corticosteroids, antibiotics and biologics (apart from one case because of suspected latent tuberculosis). However, no data exists regarding the initiation of steroid, biological (prior to CDI or after) and antibiotic therapy. Our results are in contrast with a US study reporting lack of association between the operative management and current steroids use among hospitalized patients with CDI, as well as no potential role of steroids in the treatment of CDI, although corticosteroids are a mainstay in the treatment of IBD (28).

A European study of 155 IBD patients hospitalized with CDI estimated the effects of combination antibiotic and immunomodulator therapy compared to antibiotics alone. Antibiotics and immunomodulators were associated with worse outcomes among IBD patients, higher morbidity and mortality compared to antibiotic monotherapy $(29,30)$.

In the setting of acute CDI in IBD patients, most recent AGA practice guidelines suggest postponing escalation of corticosteroids until 72 to $96 \mathrm{~h}$ after the initiation of appropriate antibiotic regimen (31). Additionally, in IBD patients with concomitant CDI, recent CDI guidelines suggest maintaining, but not escalating, existing immunosuppressive therapy, including immunomodulators such as methotrexate, azathioprine and biologic agents (32).

Also, our study highlights nonoccurrence of CMV coinfection in IBD patients with CDI and among them one $(7,1 \%)$ patient with CDI recurrence had a negative CMV biopsy.

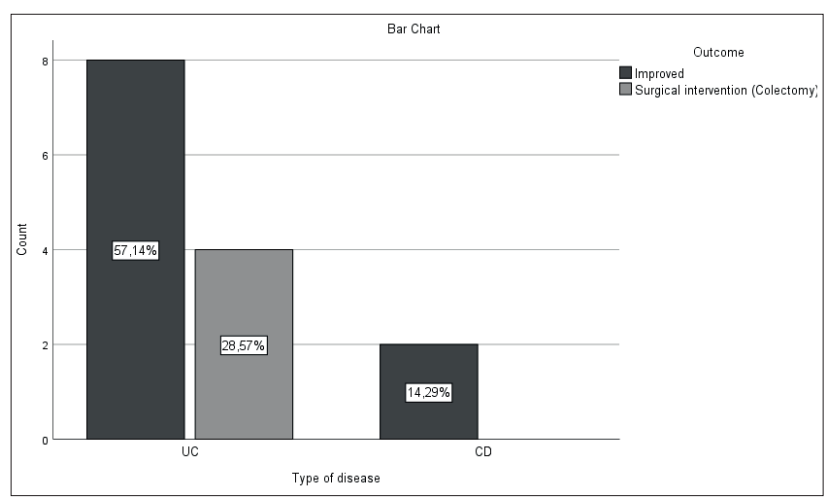

Figure 2. Clostridium difficile infection outcome

Conversely, other studies revealed a higher rate of CMV coinfection in IBD patients with CDI and the association with poor outcomes $(33,34)$.

Furthermore, the distinguishing of CDI from CMV infection may confound the clinical picture, delaying diagnosis and following treatment, resulting in high rates of colectomy $(35,36)$.

Finally, IBD and the treatment strategy (often intensive, double immunosuppression) leads to an improved therapeutic success, while also increases the risk for infectious complications and especially for CDI, as well as CMV infection $(37,38)$. The early detection of this complication in the immunocompromised patient is often more difficult due to the similar symptoms regarding intestinal infectious complications common for a flare of the underlying disease, which is consistent with other studies (39).

\section{Limitations of the study}

The strength of this study was that consecutive patients were engaged prospectively, while microbiological tests and a wide variety of demographics and medications were included. Thus, the prevalence of CDI in hospitalized patients with IBD was quite more precise.

Nevertheless, there were several limitations to this study. First, the sample size of our population was small. Second, it is uncertain that patients with positive CDI results have CDI or are sim $\neg$ ply colonized; therefore it is difficult to distinguish between CDI and a flare of IBD.

In addition, only one academic tertiary teaching hospital participated in this study and the patients attending this hospital tend to be more severely ill than subjects from smaller hospitals. However, despite these limitations, this study shows important data on the prevalence and clinical impact of CDI suspected CMV infection in patients with IBD.

\section{CONCLUSION}

Immunosuppressive medications, especially when used in combination, and older age are associated with increased risk of CDI and poor outcome. This underlines the importance of adherence to guidelines for their prevention and management.

Although, CMV is a rare colonic pathogen in the immunocompetent patient, it should be included and screened when exacerbation of IBD occurs, in patients receiving any type of immunosuppressive therapy.

More data is needed on the impact that increased patho- 
genic Th17 responses will have on IBD patients, targeting these cells may ameliorate IBD symptoms and reduce their risk of developing severe CDI.

Author's contribution: Each author gave substantial contribution to the conception or design of the work and in the acquisition, analysis and interpretation of data for the work. Each author had role in drafting the work and revising it critically for important intellectual content. Each author gave final approval of the version to be published and they agree to be accountable for all aspects of the work in ensuring that questions related to the accuracy or integrity of any part of the work are appropriately investigated and resolved.

- Conflict of interest: None declared.

- Financial support and sponsorship: Nil.

\section{REFERENCES}

1. Colombe JF, Shin A, Gibson PR. AGA clinical practice update on functional gastrointestinal symptoms in patients with inflammatory bowel disease: Expert review. Clinical Gastroenterology and Hepatology. 2019; 17(3): 380-390.

2. Maaser C, Sturm A, Vavricka SR, et al. ECCO-ESGAR Guideline for Diagnostic Assessment in IBD Part 1: Initial diagnosis, monitoring of known IBD, detection of complications. Journal of Crohn's and Colitis. 2018; 13(2): 144-164K.

3. Kim DH, Cheon JH. Pathogenesis of inflammatory bowel disease and recent advances in biologic therapies. Immune network. 2017; 17(1): 25-40.

4. Su HJ, Chiu YT, Chiu CT, et al. Inflammatory bowel disease and its treatment in 2018: Global and Taiwanese status updates. Journal of the Formosan Medical Association. 2019; 118(7): 1083-1092.

5. Hourigan SK, Oliva-Hemker M, Hutfless S. The prevalence of Clostridium difficile infection in pediatric and adult patients with inflammatory bowel disease. Digestive diseases and sciences. 2014; 59(9): 2222-2227.

6. Shoaei $\mathrm{P}$, Shojaei $\mathrm{H}$, Jalali $\mathrm{M}$, et al. Clostridium difficile isolated from faecal samples in patients with ulcerative colitis. BMC infectious diseases. 2019; 19(1): 361.

7. Curry SR. Clostridium difficile. Clin Lab Med. 2010; 30(1): 329-342.

8. Lamps LW. Infectious disorders of the GI tract. In Surgical Pathology of the GI Tract, Liver, Biliary Tract and Pancreas. 2nd ed. Philadelphia PA: Saunders Elsevier. 2009: 51-79.

9. Rodemann JF, Dubberke ER, Reske KA, Seo DH, Stone CD. Incidence of Clostridium difficile infection in inflammatory bowel disease. Clinical Gastroenterology and Hepatology. 2007; 5(3): 339-344.

10. Issa M, Vijayapal A, Graham MB, et al. Impact of Clostridium difficile on inflammatory bowel disease. Clinical Gastroenterology and Hepatology. 2007; 5(3): 345-351.

11. Kariv R, Navaneethan U, Venkatesh PG, Lopez R, Shen B. Impact of Clostridium difficile infection in patients with ulcerative colitis. Journal of Crohn's and Colitis. 2011; 5(1): 34-40.

12. Saidel-Odes L, Borer A, Odes S. Clostridium difficile in $\neg$ fection in patients with inflammatory bowel disease. Ann Gastroenterol. 2011 24: 263-270.

13. Joo H, Cho YS, Choi MG. P173 Clostridium difficile and cytomegalovirus colitis co-infection in patients with steroid-refractory ulcerative colitis. Gastroenterology. 2018; 154(1): S93.

14. McCurdy JD, Enders FT, Khanna S, et al. Increased rates of Clostridium difficile infection and poor outcomes in patients with IBD with cytomegalovirus. Inflammatory bowel diseases. 2016; 22(11): 2688-2693.

15. Chan KS, Lee WY, Yu WL. Coexisting cytomegalovirus infection in immunocompetent patients with Clostridium difficile colitis. J Microbiol Immunol Infect. 2016; 49: 829-836.

16. Saleh MM, Frisbee AL, Leslie JL, et al. Colitis-induced Th17 cells increase the risk for severe subsequent Clostridium difficile Infection. Cell host \& microbe. 2019; 25(5): 756-765.

17. Bien J, Palagani V, Bozko P. The intestinal microbiota dysbiosis and Clostridium difficile infection: is there a relationship with inflammatory bowel disease?. Therapeutic advances in gastroenterology. 2013; 6(1) 53-68.

18. Sinh P, Barrett TA, Yun L. Clostridium difficile infection and in flammatory bowel disease: a review. Gastroenterology research and practice. 2011;11pages.

19. Rahier JF, Magro F, Abreu C, et al. Second European evidence-based consensus on the prevention, diagnosis and management of opportunistic infections in inflammatory bowel disease. Journal of Crohn's and Colitis. 2014; 8(6): 443-468.

20. Kandiel A, Lashner B. Cytomegalovirus colitis complicating inflammatory bowel disease. American Journal of Gastroenterology. 2006; 101(12): 2857-2865.

21. Joo H, Cho YS, Choi MG. P173 Clostridium difficile and cytomegalovirus colitis co-infection in patients with steroid-refractory ulcerative colitis. Inflammatory Bowel Diseases. 2018; 24(1): S65.

22. Xu H, Tang H, Xu T, et al. Retrospective analysis of Clostridium difficile infection in patients with ulcerative colitis in a tertiary hospital in China. BMC gastroenterology. 2019); 19(1): 3.

23. Centers for Control Disease and Prevention. Retrieved from: https:// www.cdc.gov/cdiff/clinicians/faq.html

24. Regnault H, Bourrier A, Lalande V, et al. Prevalence and risk factors of Clostridium difficile infection in patients hospitalized for flare of inflammatory bowel disease: a retrospective assessment. Digestive and Liver Disease. 2014; 46(12): 1086-1092.

25. D'Aoust J, Battat R, Bessissow T. Management of inflammatory bowel disease with Clostridium difficile infection. World journal of gastroenterology. 2017; 23(27): 4986.

26. Razik R, Rumman A, Bahreini Z, McGeer A, Nguyen GC. Recurrence of Clostridium difficile infection in patients with inflammatory bowel disease: the RECIDIVISM study. The American journal of gastroenterology. 2016; 111(8): 1141.

27. Horton HA, Dezfoli S, Berel D, et al. Antibiotics for treatment of Clostridium difficile infection in hospitalized patients with inflammatory bowel disease. Antimicrobial agents and chemotherapy. 2014;58(9):5054-5059.

28. Dirweesh A, Alvarez C, Khan M, et al. Lack of Association Between the Clinical Outcome of Clostridium difficile Infection and Current Steroids Use. Gastroenterology research. 2017;10(2):116.

29. Ben-Horin S, Margalit M, Bossuyt P, et al. Combination immunomodulator and antibiotic treatment in patients with inflammatory bowel disease and Clostridium difficile infection. Clinical Gastroenterology and Hepatology. 2009; 7(9): 981-987.

30. Ananthakrishnan AN, McGinley EL, Saeian K, Binion DG. Temporal trends in disease outcomes related to Clostridium difficile infection in patients with inflammatory bowel disease. Inflammatory bowel diseases. 2010; 17(4): 976-983.

31. Khanna S, Shin A, Kelly CP. Management of Clostridium difficile infection in inflammatory bowel disease: expert review from the clinical practice updates committee of the AGA institute. Clinical Gastroenterology and Hepatology. 2017; 15(2): 166-174.

32. Surawicz CM, Brandt LJ, Binion DG, et al. Guidelines for diagnosis, treatment, and prevention of Clostridium difficile infections. Am J Gastroenterol. 2013; 108(4): 478-498.

33. Chan KS, Lee WY, Yu WL. Coexisting cytomegalovirus infection in immunocompetent patients with Clostridium difficile colitis. J Microbiol Immunol Infect. 2016; 49(6): 829-836.

34. Li Y, Xu H, Xu T, et al. Case-Control Study of Inflammatory Bowel Disease Patients with and without Clostridium difficile Infection and Poor Outcomes in Patients Coinfected with C. difficile and Cytomegalovirus. Digestive Diseases and Sciences. 2018; 63: 3074-3083.

35. Florescu DF, Mindru C, Chambers HE, Kalil AC. Clostridium difficile and cytomegalovirus colitis co-infection: search for the hidden 'bug'. Transplant Infectious Disease. 2011; 13(4): 411-415.

36. Kurtz M, Morgan M. Concomitant Clostridium difficile colitis and cytomegalovirus colitis in an immunocompetent elderly female. BMJ case reports. 2012: bcr2012007273.

37. Kucharzik T, Maaser C. Infections and chronic inflammatory bowel disease. Visceral Medicine. 2014; 30(5): 326-332.

38. Nannegari V, Friedman M, Green J, MacDermott R. Simultaneous Cytomegalovirus and Clostridium difficile Colitis in a Patient with Inflammatory Bowel Disease: 960. American Journal of Gastroenterology. 2011; 106.

39. Ford AC, Peyrin-Biroulet L. Opportunistic infections with antitumor necrosis factor- $\alpha$ therapy in inflammatory bowel disease: meta-analysis of randomized controlled trials. American Journal of Gastroenterology. 2013); 108(8): 1268-1276. 